University of Nebraska - Lincoln

DigitalCommons@University of Nebraska - Lincoln

January 1980

\title{
Purification, growth, structure, optical and electrical properties of single crystals of the $\pi$-molecular complex of phenothiazine with pyromellitic dianhydride
}

\author{
R. Anthonj \\ Physikalisches Institut-Teil 3 der Universität Stuttgart \\ N. Karl \\ Physikalisches Institut-Teil 3 der Universität Stuttgart \\ Beverly E. Robertson \\ University of Regina, Regina, Saskatchewan \\ John J. Stezowski \\ University of Nebraska - Lincoln, jjs@unlserve.unl.edu
}

Follow this and additional works at: https://digitalcommons.unl.edu/chemistrystezowski

Part of the Chemistry Commons

Anthonj , R.; Karl, N.; Robertson, Beverly E.; and Stezowski, John J., "Purification, growth, structure, optical and electrical properties of single crystals of the $\pi$-molecular complex of phenothiazine with pyromellitic dianhydride" (1980). John J. Stezowski Publications. 5.

https://digitalcommons.unl.edu/chemistrystezowski/5

This Article is brought to you for free and open access by the Published Research - Department of Chemistry at DigitalCommons@University of Nebraska - Lincoln. It has been accepted for inclusion in John J. Stezowski Publications by an authorized administrator of DigitalCommons@University of Nebraska - Lincoln. 


\title{
Purification, growth, structure, optical, and electrical properties of single crystals of the $\pi$-molecular complex of phenothiazine with pyromellitic dianhydride
}

\author{
R. Anthonj and N. Karl \\ Physikalisches Institut-Teil 3 der Universität Stuttgart, Pfaffenwaldring 57, D 7000 Stuttgart-80, \\ Federal Republic of Germany \\ Beverly E. Robertson \\ Faculty of Science, University of Regina, Regina, Saskatchewan, Canada S4S OA2 \\ John J. Stezowski
}

Institut für Organische Chemie, Biochemie und Isotopenforschung der Universität Stuttgart, Pfaffenwaldring 55, D 7000 Stuttgart-80, Federal Republic of Germany

(Received 18 December 1978; accepted 10 July 1979)

Single crystals of the black phenothiazine:pyromellitic dianhydride (PTZ:PMDA) 1:1 donor-acceptor complex, have been grown by sublimation from the zone-refined components. The PTZ:PMDA complex crystalizes with $P \overline{1}$ symmetry $(Z=2), a=7.197(1), b=19.072(5), c=6.886(1) \AA, \alpha=84.79(1)^{\circ}$, $\beta=72.98(1)^{\circ}, \gamma=85.72(2)^{\circ}$ at $T=23^{\circ} \mathrm{C}$. The crystal structure model was refined with 5214 data $\left\{(\sin \theta / \lambda)_{\max }=0.8071 \AA^{-1}\right\}$ to give $R=0.049$ and $R_{w}=0.089$. The crystal packing consists of two polar endless ...DADA... stacks related to one another by $\overline{1}$. The packing is compared with that in the analogous anthracene:PMDA complex. The PTZ molecule displays a modest fold about the $N \ldots S$ axis (dihedral angle $176.4^{\circ}$ ), however, the deviation from planarity is small in comparison with that in PTZ crystals. The stack axis is nearly perpendicular to the molecular planes, consequently the CT-dichroism lies essentially in the principal axis system of the indicatrix. The absorption edge is not very sharp, even at $4.2 \mathrm{~K}$; it is located at $900 \pm 25 \mathrm{~nm}$. This value is compared to the ionization energy of PTZ and to the energy of the CT transition in anthracene:PMDA. No optical emission could be detected; the pathways of radiationless deactivation are discussed in terms of CT-vibron coupling. An intrinsic dark conductivity of $\sigma \sim 10^{-12} \Omega^{-1} \mathrm{~cm}^{-1}$ was found. It is consistent with the value of the band gap (1.7 eV) obtained both from the measured activation energy of the dark conductivity and from the estimated thermal dissociation of CT excitons. Possible implications of the polar stacking on the excitonic energy levels and energy transport are briefly discussed.

\section{INTRODUCTION}

Donor-acceptor (D:A) complexes of aromatic $\pi$-electron systems often possess interesting physical properties such as low energy optically allowed intermolecular electronic transitions (charge transfer, CT transitions) which in special cases even occur thermally to the extent that they may lead to metallic like conductivities ("organic metals").

Such properties are dependent not only on specific molecular properties of the individual components such as ionization energy of the donor and electron affinity of the acceptor, but are also strongly influenced by details of the relative arrangement of and interactions in the crystal lattice and by crystal purity and quality. Therefore a comprehensive investigation of these materials is best pursued as a collaborative effort by solid state physicists, crystallographers and crystal-growers.

The necessity of high purity dictates the selection of thermally stable compounds which can be zone refined and of which single crystals can be grown from their vapor or melt. With these requirements in mind we have concentrated our attention on fused 3-ring compounds containing aromatic moieties.

The red 1:1 complex between anthracene, $A$, and pyromellitic dianhydride, PMDA, was a first choice ${ }^{1}$ among a number of possible combinations between pheno- thiazine, anthracene, carbazole, fluorene, acridine, phenazine, and PMDA. This CT complex has been extensively studied and characterized. For example, photocurrent-excitation spectra and charge carrier mobilities have been measured ${ }^{2}$; piezoreflection spectra have been recorded ${ }^{3}$; zero phonon optical transitions with zero field splitting of Stark levels were observed ${ }^{4}$ and have led to a more detailed understanding of electron-phonon coupling ${ }^{5}$; the complete triplet-exciton fine structure tensor has been determined from photocurrent resonances in a magnetic field (without use of microwaves) ${ }^{6}$; and a high precision crystal structure analysis has been carried out. ${ }^{\text {? }}$

We now wish to report structural, crystallographic, optical, and electrical properties of a related system, that of the black complex between phenothiazine, PTZ, and PMDA. We believe this to be the first example of a single crystal of a D:A complex with a CT transition in the infrared region obtained from zone refined components.

Several underlying questions prompted us to study this system. Among them were questions such as how does the crystal packing of the complex with noncentrosymmetric PTZ compare with that in the A:PMDA analog in which both components occupy sites with inversion symmetry. We also wanted to examine what structural ev idence there might be for charge transfer in the ground 
state and to determine what effects the CT interactions have on the normally bent conformation of PTZ. ${ }^{8,9} \mathrm{We}$ were further interested in investigating how the $\mathrm{CT}$ absorption edge correlates with the molecular data; e.g., is there a specific site shift caused by crystal packing? We also wondered whether or not charge separation by one intermolecular separation at the optical CT transition might be a precursary step for efficient charge carrier generation. Another point of interest concerned the distinct possibility that there might be sufficient thermal excitation of the CT transition to lead to free carriers giving rise to dark conductivity.

\section{PURIFICATION, CRYSTAL GROWTH AND PRELIMINARY CRYSTALLOGRAPHIC DATA FOR PTZ : PMDA}

Phenothiazine $(99 \%$, from Schuchardt, Munich) was first purified by liquid chromatography (carried out in the dark) of a benzene solution over $\mathrm{Al}_{2} \mathrm{O}_{3}$, then by vacuum sublimation and by zone refining under 400 Torr $\mathrm{N}_{2}$ for 100 zone passages. The purified fraction resulting therefrom was further zone refined (in a new ampoule) for another 100 passages to give a light yellow colored product.

Commercial PMDA (96\%, from Fluka, Buchs, Switzerland) was first dehydrated to remove any free acid. This was effected by gentle heating under vacuum during which time the temperature was slowly raised to the melting point. If this preliminary step is not executed, the material turns black in the zone refining step as the result of the elimination of $\mathrm{CO}_{2}$ and $\mathrm{H}_{2} \mathrm{O}^{10}$; this decom-

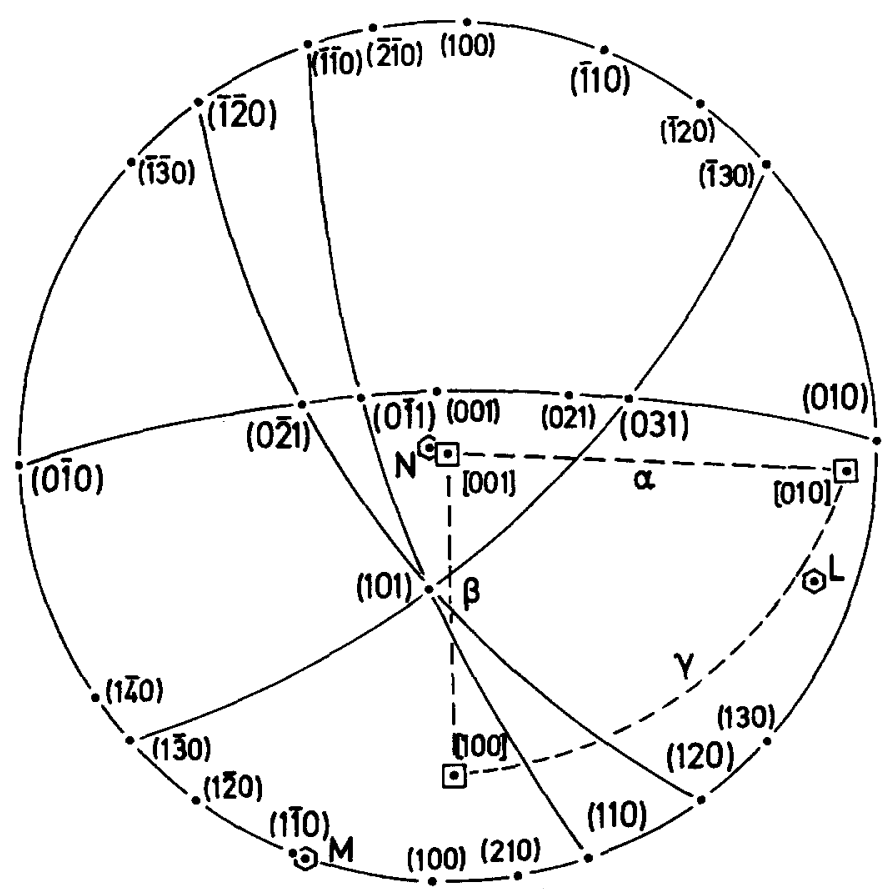

FIG. 1. Stereographic projection of crystallographic axes and face normals of PTZ: PMDA. Crystals grown by sublimation are dominated by the faces which are presented in larger numbers. In addition the molecular axes of PMDA, $L$ (long axis), $M$ (medium axis), $N$ (normal to central phenyl ring) are indicated.

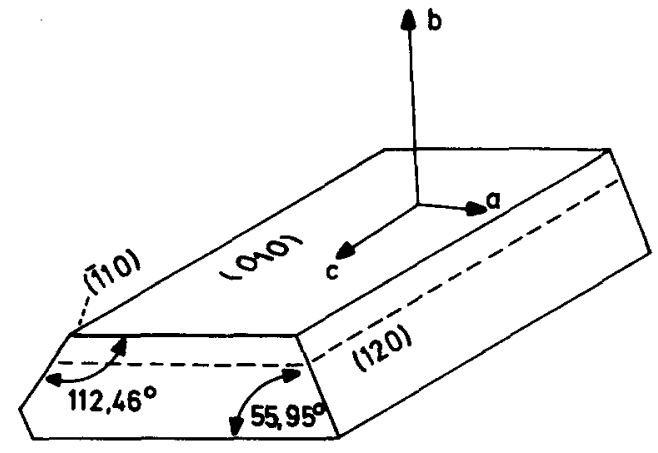

FIG. 2. Orientation and angles of typical PTZ: PMDA sublimation crystals. The cleavage plane is indicated.

position process eventually results in explosions. Zone refined PMDA is colorless and transparent but turns milky white if not protected from humidity.

The plate sublimation method was used to obtain single crystals of the PTZ:PMDA complex. This is a newly developed technique ${ }^{11}$ which typically yields a small number of freely grown crystals with well formed faces. This method has proven to be especially well suited for the growth of crystals of DA-complexes because it utilizes nearly isothermal conditions which serves to minimize the segregation of individual donor or acceptor phases. The components ( $2 \mathrm{~g}$ in molar ration $1: 1$ ) were sealed in evacuated pyrex ampoules. Crystals were grown over a period of several weeks with the system maintained at $150^{\circ} \mathrm{C}$ with a temperature gradient of $1 \% / 4 \mathrm{~cm}$. Lustrous black crystals with dimensions up to $10 \times 5 \times 2 \mathrm{~mm}^{3}$ were so obtained. The complex melts with slow decomposition at $174(2)^{\circ}$ which prevents zone refining of the combined components.

The crystals show a propensity for cleavage with (010) as the cleavage plane, see Fig. 2. Very thin cleaved sheets are greenish in color and strongly dichroitic. Elongated crystal platelets of the same orientation but only a few $\mu \mathrm{m}$ thick were prepared by cooling solutions of the complex in dryed xylene at a rate of $10^{\circ} \mathrm{h}^{-1}$.

We obtained what may be a modification of the PTZ:PMDA complex from sublimation at higher temperature $\left(163^{\circ}\right)$. Thin layers of the crystals so grown are brownish in color, show a much less pronounced dichroism and display no cleavage tendency.

An initial set of lattice parameters for the first modification of PTZ:PMDA was obtained by one of us (R.A.) in collaboration with $\mathrm{H}$. Schulz and K. H. Thiemann at the Max-Planck-Institut für Festkörperforschung in Stuttgart. Crystal morphology was examined with a 2-circle optical goniometer; the results are presented in Figs. 1 and 2. The crystals are triclinic (final lattice parameters are given below). The unit cell volume and specific gravity determined by the flotation procedure, $1.550(5) \mathrm{g} \mathrm{cm}^{-3}$, are indicative of a $1: 1$ stoichiometry with $Z=2$. Elemental analysis was consistent with this result: $\mathrm{C}=62.97 \%$ (calc 63.31 ) $\mathrm{H}=2.79 \%$ (2. 66), and $\mathrm{N}=3.24 \%(3.36)$. 


\section{STRUCTURE DETERMINATION AND REFINEMENT}

An irregularly shaped crystal with approximate dimensions $0.50 \times 0.50 \times 0.80 \mathrm{~mm}^{3}$ and bounded by nine lattice planes was used for data collection with a Syntex $P \overline{1}$ autodiffractometer. The following lattice parameters were determined by least squares refinement ${ }^{12}$ with automatically centered $2 \theta$ values of 45 reflections in the angular range $39<2 \theta<45^{\circ}\left(\mathrm{MoK}_{\alpha}, \lambda=0.71069 \AA\right)$ : $a=7.197(1), b=19.072(5), c=6.886(1) \AA, \alpha=84.79(1)$, $\beta=72.98(1), \gamma=85.72(2)^{\circ}$ at $T=23^{\circ} \mathrm{C}$; the statistics were strongly centrosymmetric therefore space group $\mathrm{P} \overline{1}$ was selected and proved appropriate in subsequent refinement.

Diffraction intensities were measured in a $\omega$-scan mode for which the scan rate was allowed to vary as a function of maximum peak intensity from $2.0^{\circ}$ to $24.0^{\circ}$ $\min ^{-1}$; the scan range was $1.0^{\circ}$ and background intensity was measured on each side of the reflection, $\Delta \omega=1.0^{\circ}$, for one half the total scan time. Of the 7019 unique reflections so measured ( $\sin \theta / \lambda \leq 0.8071 \AA^{-1}$, monochromatized $\mathrm{MoK}_{\alpha}$ radiation), 5216 were classified as objectively observed $[I \geq 3 \sigma(I)]$ and used in subsequent refinement. Three reference reflections, monitored after each 200 data were measured, displayed a similar systematic decrease in their intensity totalling $\sim 8 \%$. It was not possible to determine the cause of this decrease but the data were subsequently corrected for it and for Lorentz and polarization effects. Trial absorption correction calculations $\left(\mu=2.27 \mathrm{~cm}^{-1}\right)$ demonstrated the associated errors to be between $1.7 \%$ and $2.1 \%$; corection was deemed to be unnecessary.

The initial structural model was obtained by direct methods and was refined by full-matrix least-squares. The final cycles of refinement were carried out with the program ORFLS. ${ }^{13}$ The $\mathrm{C}, \mathrm{N}, \mathrm{O}$, and $\mathrm{S}$ atoms were refined with anisotropic temperature factors to an $R$ value of 0.075 after which the $\mathrm{H}$ atoms were placed in calculated positions. The $\mathrm{N}$-hydrogen atom was assumed to be "intra" as predicted by Malrieu and Pullman" ${ }^{14}$ that is, it was assumed to be on the opposite side of the planes of the phenyl groups than the $\mathrm{N}$ and $\mathrm{S}$ atoms. All position parameters, anisotropic temperature factors for $\mathrm{C}, \mathrm{N}, \mathrm{O}$, and $\mathrm{S}$ atoms, isotropic temperature factors for $\mathrm{H}$ atoms and a scale factor were refined.

The refinement of $\mathrm{C}, \mathrm{N}, \mathrm{O}$, and $\mathrm{S}$ parameters was generally routine as was that for most $\mathrm{H}$ atoms. Atoms $H(28)$ and $H(29)$ behaved somewhat erratically which most likely reflects the thermal motion in this portion of the $\mathrm{PTZ}$ molecule. Atom $\mathrm{H}(20)$, that bonded to the $\mathrm{N}$ atom, moved to a position approximately $0.50 \AA$ from the latter atom. This atom was then removed from the refinement and a difference Fourier map was calculated. The map contained a large asymmetric peak in the position to which atom $\mathrm{H}(20)$ refined; one attribute of the asy $\mathrm{m}-$ metry was a shoulder further removed from atom $N(20)$. Subtraction of a symmetrical core from this peak leaves weak electron density associated with this shoulder that is in the expected position for an $\mathrm{H}$ atom in the intra position.
The refinement of the model [less atom $\mathrm{H}(20)$ ] was continued to convergence (shifts/errors $<0.06$ ). The weight of an individual reflection was calculated to be $w=\left(0.0580+0.05835\left|F_{0}\right|+0.000018\left|F_{0}\right|^{2}\right)^{-2}$. The resultant conventional residuals for the contributing reflections $^{15}$ are $R=0.049, R_{w}=0.089, S=1.19$. Because of program limitations, all parameters could not be refined simultaneously. The selection of parameters refined in each successive cycle was altered so that all correlations between variables were included at some point during the final cycles of least-squares. Two very

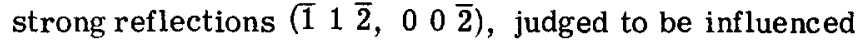
by secondary extinction, were excluded from the refinement. An extinction correction was not applied because of the irregular morphology of the crystals. The final coordinates and thermal parameters are given in Table I.

\section{DISCUSSION OF THE CRYSTAL STRUCTURE}

The bond lengths and angles for the two molecular components of PTZ :PMDA are presented in Figs. 3(a)-(d). Crystals of PTZ : PMDA consist of the usual columns of alternating donor and acceptor molecules. The crystal packing is illustrated in steroscopic projections $^{16}$ in Figs. 4 and 5 . The stack axis is parallel to the crystallographic $c$ - axis.

The crystal packing in PTZ:PMDA both resembles and contrasts with that in A:PMDA. ${ }^{7}$ Of particular interest is the comparison of the intrastack acceptor-donoracceptor arrangement in the two structures, Fig. 6. They are very similar with respect to the position of the donor and one acceptor molecule but not to that of the second acceptor. The A molecule of the A:PMDA complex is constrained by crystallographic symmetry to having a centrosymmetric immediate environment whereas the PTZ molecule of PTZ : PMDA is not and does not. The A molecule is nearly planar while the PTZ molecule displays a fold about the line defined by the $S \rightarrow N$ vector; see Fig. 6 . We believe the differences in packing reflect the less symmetrical chemical structure of PTZ and its better donor character. These points may increase the importance of charge distribution in the crystal packing of the PTZ: PMDA complex.

The crystal packing of the A : PMDA complex presents identical environments for both ends of the $A$ and PMDA molecules but allows differences in charge distribution in the two carbonyl $\mathrm{C}-\mathrm{O}$ bonds of the symmetry unique anhydride ring. The evidence indicating that such differences occur was described. ${ }^{7}$ Briefly, the exocyclic $\mathrm{C}-\mathrm{O}$ bond lengthens, [see $\mathrm{C}(8)-\mathrm{O}(13)$ in Fig. $3(\mathrm{c})]$, as the negative charge on its oxygen atom increases and an accompanying shortening of the adjacent endocyclic $\mathrm{C}-\mathrm{O}$ bond length [e.g., $\mathrm{C}(8)-\mathrm{O}(12)]$ occurs. The suitability of the oxygen atom of a specific carbonyl group to serve as a carrier for negative charge is dependent on the contact distances between it and other charge carrying oxygen atoms. To assess this environmental factor for PTZ : PMDA we calculated $\Sigma 1 / r^{2}$ for all carbonyl $O$. . O contacts within $6.0 \AA$; the values are $\left[O(11), 0.27 \AA^{-2}\right],[O(13), 0.23],[O(14), 0.41]$, and $[O(16), 0.37]$. From the charge repulsion standpoint, the $O(11) \ldots O(13)$ end of the PMDA molecule is clearly 
TABLE I. Positional and thermal parameters with standard deviations.

A. Positional parameters of carbon, oxygen, sulfur, and nitrogen atoms $\left(\times 10^{4}\right)$.

\begin{tabular}{|c|c|c|c|}
\hline & $x / a$ & $y / b$ & $z / c$ \\
\hline$C(1)$ & $4636(2)$ & $2555(1)$ & $1773(2)$ \\
\hline$C(2)$ & $3096(2)$ & $2118(1)$ & $2165(2)$ \\
\hline$C(3)$ & $1156(2)$ & $2369(1)$ & $2698(2)$ \\
\hline $\mathrm{C}(4)$ & $597(2)$ & $3078(1)$ & $2845(2)$ \\
\hline$C(5)$ & $2140(2)$ & $3515(1)$ & $2443(2)$ \\
\hline$C(6)$ & $4076(2)$ & $3267(1)$ & $1938(2)$ \\
\hline$C(7)$ & $3110(2)$ & $1348(1)$ & $2070(2)$ \\
\hline$C(8)$ & $-66(2)$ & $1753(1)$ & $3031(2)$ \\
\hline $\mathrm{C}(9)$ & $2124(2)$ & $4293(1)$ & $2433(2)$ \\
\hline $\mathrm{C}(10)$ & $5296(2)$ & $3884(1)$ & $1625(2)$ \\
\hline$O(11)$ & $4424(2)$ & $918(1)$ & $1620(2)$ \\
\hline $\mathrm{O}(12)$ & $1166(2)$ & $1162(1)$ & $2607(2)$ \\
\hline$O(13)$ & $-1799(2)$ & $1717(1)$ & $3567(2)$ \\
\hline$O(14)$ & $809(2)$ & $4719(1)$ & $2773(2)$ \\
\hline$O(15)$ & $4047(2)$ & $4479(1)$ & $1945(2)$ \\
\hline$O(16)$ & $7020(2)$ & $3919(1)$ & $1190(2)$ \\
\hline$S(17)$ & $198.9(5)$ & $7419.9(2)$ & $1789.6(6)$ \\
\hline$C(18)$ & $-1914(2)$ & $6938(1)$ & $2459(2)$ \\
\hline $\mathrm{C}(19)$ & $-3794(2)$ & $7259(1)$ & $3017(2)$ \\
\hline$N(20)$ & $-4141(2)$ & $7991(1)$ & $3106(2)$ \\
\hline$C(21)$ & $-2709(2)$ & $8480(1)$ & $2844(2)$ \\
\hline$C(22)$ & $-716(2)$ & $8287(1)$ & $2263(2)$ \\
\hline $\mathrm{C}(23)$ & $-1659(2)$ & $6208(1)$ & $2389(2)$ \\
\hline $\mathrm{C}(24)$ & $-3248(3)$ & $5789(1)$ & $2840(3)$ \\
\hline $\mathrm{C}(25)$ & $-5116(3)$ & $6107(1)$ & $3370(2)$ \\
\hline$C(26)$ & $-5369(2)$ & $6832(1)$ & $3469(2)$ \\
\hline$C(27)$ & $-3289(4)$ & $9188(1)$ & $3128(3)$ \\
\hline $\mathrm{C}(28)$ & $-1933(5)$ & $9688(1)$ & $2857(4)$ \\
\hline$C(29)$ & $34(5)$ & $9496(1)$ & $2286(4)$ \\
\hline $\mathrm{C}(30)$ & $637(3)$ & $8800(1)$ & $1980(3)$ \\
\hline
\end{tabular}

B. Thermal parameters $\left(U_{t \xi}\right)^{\mathrm{a}}$ of carbon, oxygen, sulfur, and nitrogen atoms $\left(\times 10^{4}\right)$.

\begin{tabular}{|c|c|c|c|c|c|c|}
\hline & $U_{11}$ & $U_{22}$ & $U_{33}$ & $U_{12}$ & $U_{13}$ & $U_{23}$ \\
\hline$C(1)$ & $323(5)$ & $481(6)$ & $438(5)$ & $-1(4)$ & $-89(4)$ & $-34(4)$ \\
\hline$C(2)$ & $366(5)$ & $405(5)$ & $376(5)$ & $3(4)$ & $-98(4)$ & $-12(4)$ \\
\hline$C(3)$ & $346(5)$ & $426(5)$ & $384(5)$ & $-38(4)$ & $-101(4)$ & $18(4)$ \\
\hline$C(4)$ & $325(5)$ & $453(6)$ & $472(6)$ & $16(4)$ & $-97(4)$ & $3(4)$ \\
\hline $\mathrm{C}(5)$ & $382(5)$ & $403(5)$ & $385(5)$ & $-8(4)$ & $-103(4)$ & 1(4) \\
\hline$C(6)$ & $354(5)$ & $456(5)$ & $367(5)$ & $-45(4)$ & $-89(4)$ & $-9(4)$ \\
\hline$C(7)$ & $514(7)$ & $434(6)$ & $494(6)$ & $9(5)$ & $-133(5)$ & $-33(5)$ \\
\hline $\mathrm{C}(8)$ & $433(6)$ & $494(6)$ & $500(6)$ & $-109(5)$ & $-135(5)$ & $40(5)$ \\
\hline$C(9)$ & $572(8)$ & $415(6)$ & $542(7)$ & $0(5)$ & $-151(6)$ & $-20(5)$ \\
\hline$C(10)$ & $473(7)$ & $522(7)$ & $473(6)$ & $-124(5)$ & $-131(5)$ & $-17(5)$ \\
\hline$O(11)$ & $702(8)$ & $504(6)$ & $880(9)$ & $143(6)$ & $-168(7)$ & $-119(6)$ \\
\hline$O(12)$ & $577(6)$ & $440(5)$ & $646(6)$ & $-94(4)$ & $-171(5)$ & $-16(4)$ \\
\hline$O(13)$ & $438(6)$ & $696(7)$ & $766(8)$ & $-191(5)$ & $-154(5)$ & $86(6)$ \\
\hline$O(14)$ & $729(9)$ & $479(6)$ & $977(10)$ & $153(6)$ & $-205(8)$ & $-80(6)$ \\
\hline$O(15)$ & $617(7)$ & $450(5)$ & $632(6)$ & $-111(5)$ & $-173(5)$ & $-26(4)$ \\
\hline$O(16)$ & $477(6)$ & $748(8)$ & $785(8)$ & $-213(6)$ & $-150(6)$ & $-18(6)$ \\
\hline$S(17)$ & $324(2)$ & $532(2)$ & $619(2)$ & $-108(1)$ & $-97(1)$ & $-142(2)$ \\
\hline$C(18)$ & $357(5)$ & $442(5)$ & $356(5)$ & $4(4)$ & $-103(4)$ & $-31(4)$ \\
\hline$C(19)$ & $336(5)$ & $545(6)$ & $371(5)$ & $-4(4)$ & $-108(4)$ & $-27(4)$ \\
\hline $\mathrm{N}(20)$ & $404(6)$ & $550(7)$ & $757(9)$ & $75(5)$ & $-135(6)$ & $-122(6)$ \\
\hline$C(21)$ & $545(7)$ & $468(6)$ & $418(6)$ & $56(5)$ & $-131(5)$ & $-32(5)$ \\
\hline$C(22)$ & $492(7)$ & $471(6)$ & $391(5)$ & $-47(5)$ & $-127(5)$ & $-7(4)$ \\
\hline$C(23)$ & $518(7)$ & $446(6)$ & $523(7)$ & $3(5)$ & $-124(6)$ & $-8(5)$ \\
\hline$C(24)$ & $737(11)$ & $500(7)$ & $594(8)$ & $-138(7)$ & $-188(8)$ & $35(6)$ \\
\hline$C(25)$ & $605(9)$ & $725(10)$ & $537(8)$ & $-273(8)$ & $-163(7)$ & $74(7)$ \\
\hline$C(26)$ & $382(6)$ & $764(10)$ & $484(7)$ & $-98(6)$ & $-114(5)$ & $-3(6)$ \\
\hline$C(27)$ & $933(15)$ & $501(8)$ & $654(10)$ & $202(9)$ & $-180(10)$ & $-57(7)$ \\
\hline $\mathrm{C}(28)$ & $1286(24)$ & $451(8)$ & $844(14)$ & $30(11)$ & $-237(14)$ & $-28(9)$ \\
\hline$C(29)$ & $1247(24)$ & $500(9)$ & $871(15)$ & $-261(12)$ & $-236(14)$ & $47(9)$ \\
\hline$C(30)$ & $702(11)$ & $574(9)$ & $642(9)$ & $0196(8)$ & $-147(8)$ & $30(7)$ \\
\hline
\end{tabular}


TABLE I (Continued)

C. Positional and thermal parameters for the hydrogen atoms $\left(\times 10^{3}\right)$. Hydrogen atoms are assigned the same number as the carbon atom to which they are bonded.

\begin{tabular}{lrrlr} 
& \multicolumn{1}{c}{$x / a$} & \multicolumn{1}{c}{$y / b$} & \multicolumn{1}{l}{$z / c$} & \multicolumn{1}{l}{} \\
$\mathrm{H}(1)$ & $597(3)$ & $230(1)$ & $153(3)$ & $71(6)$ \\
$\mathrm{H}(4)$ & $-84(3)$ & $327(1)$ & $320(3)$ & $60(5)$ \\
$\mathrm{H}(23)$ & $-38(4)$ & $595(1)$ & $186(3)$ & $77(7)$ \\
$\mathrm{H}(24)$ & $-299(4)$ & $528(1)$ & $280(4)$ & $82(7)$ \\
$\mathrm{H}(25)$ & $-624(4)$ & $582(1)$ & $381(4)$ & $90(8)$ \\
$\mathrm{H}(26)$ & $-669(4)$ & $711(1)$ & $369(4)$ & $88(7)$ \\
$\mathrm{H}(27)$ & $-451(4)$ & $925(2)$ & $344(4)$ & $97(9)$ \\
$\mathrm{H}(28)$ & $-268(4)$ & $1020(1)$ & $328(4)$ & $92(8)$ \\
$\mathrm{H}(29)$ & $64(5)$ & $989(2)$ & $212(5)$ & $125(11)$ \\
$\mathrm{H}(30)$ & $195(4)$ & $867(1)$ & $161(4)$ & $100(9)$ \\
\hline
\end{tabular}

a Of form $\exp \left[-2 \pi^{2}\left(a^{* 2} h^{2} U_{11}+b^{* 2} k^{2} U_{22}+c^{* 2} l^{2} U_{33}+2 a^{*} b^{*} h k U_{12}+2 a^{*} c^{*} h l U_{13}+2 b^{*} c^{*} k l U_{23}\right)\right]$.

favored to be that with the greater negative charge density. Negative charge on atom $\mathrm{O}(13)$ is further favored by a weak interstack hydrogen bond to a $\mathrm{N}-\mathrm{H}$ moiety, $\mathrm{O}(13) . . \mathrm{N}=3.189(2) \AA$.

The charge separation in the PMDA molecule appears to influence the conformation of the PTZ molecule. The fold mentioned earlier (see Fig. 6) is consistent with a phenyl ring being drawn toward the $O(11), \ldots O(13)$ and of the PMDA molecule above it. The associated intramolecular dipole becomes an interstack quadrupole through the inversion operator at $0,0,1 / 2$ of the unit cell, (Figs. 4, 5). The PMDA molecule also displays significant deviation from planarity, Fig. 7.

The observation of the peak displaced 0.50 A from the $\mathrm{N}$ atom implies that the conformational enantiomer (mirror image) of PTZ occasionally occupies the site of the PTZ molecule. The peak height $\left(\sim 1 / 3\right.$ of an $\left.e^{-}\right)$implies that the exchange occurs in at least $2 \%$ of the PTZ
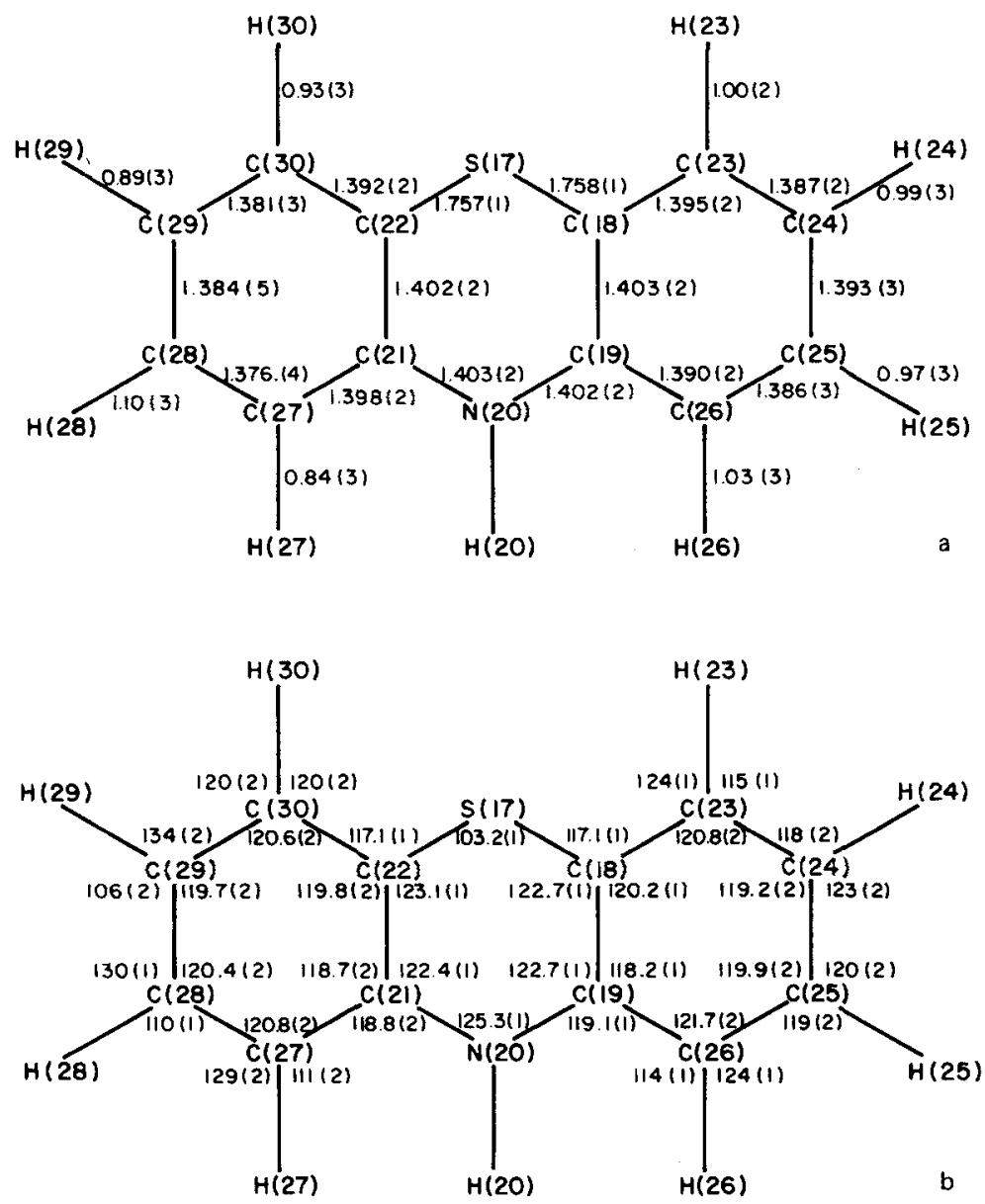
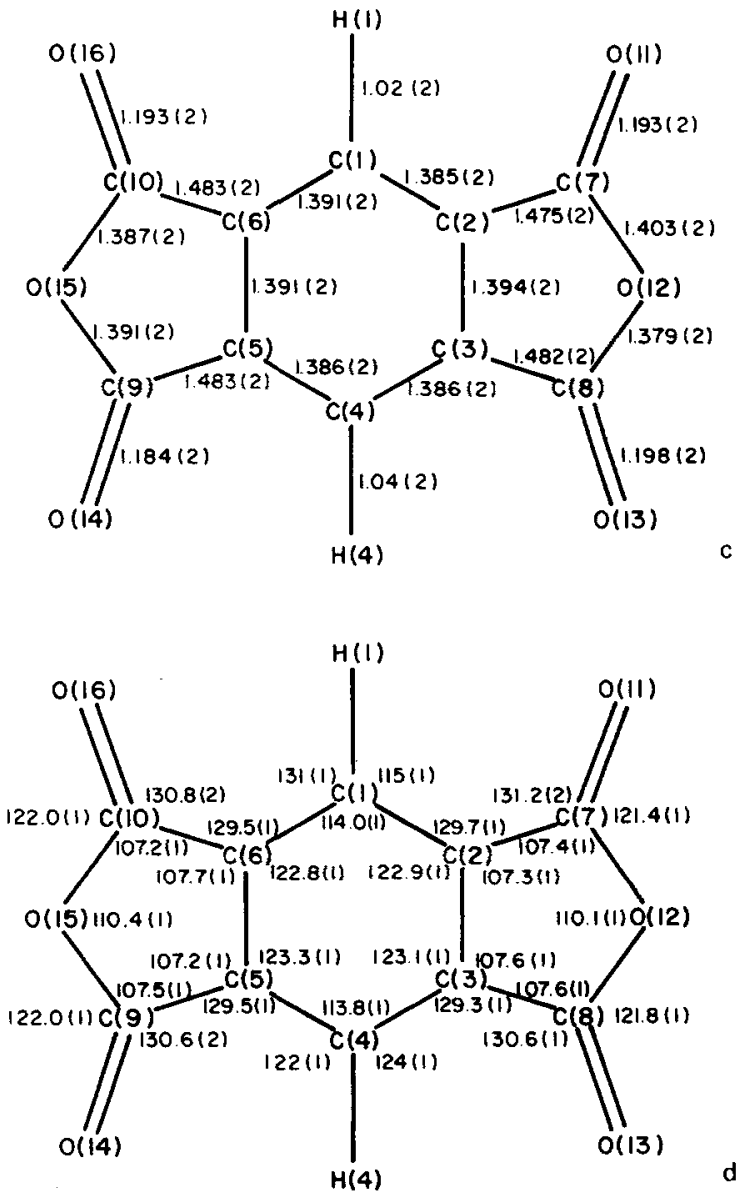

FIG. 3. Atom labeling, bond lengths $(\AA)$, and angles $\left(^{\circ}\right)$ in PTZ: PMDA; (a) bond lengths for PTZ, (b) bond angles for PTZ, (c) bond lengths for PMDA, (d) bond angles for PMDA. 


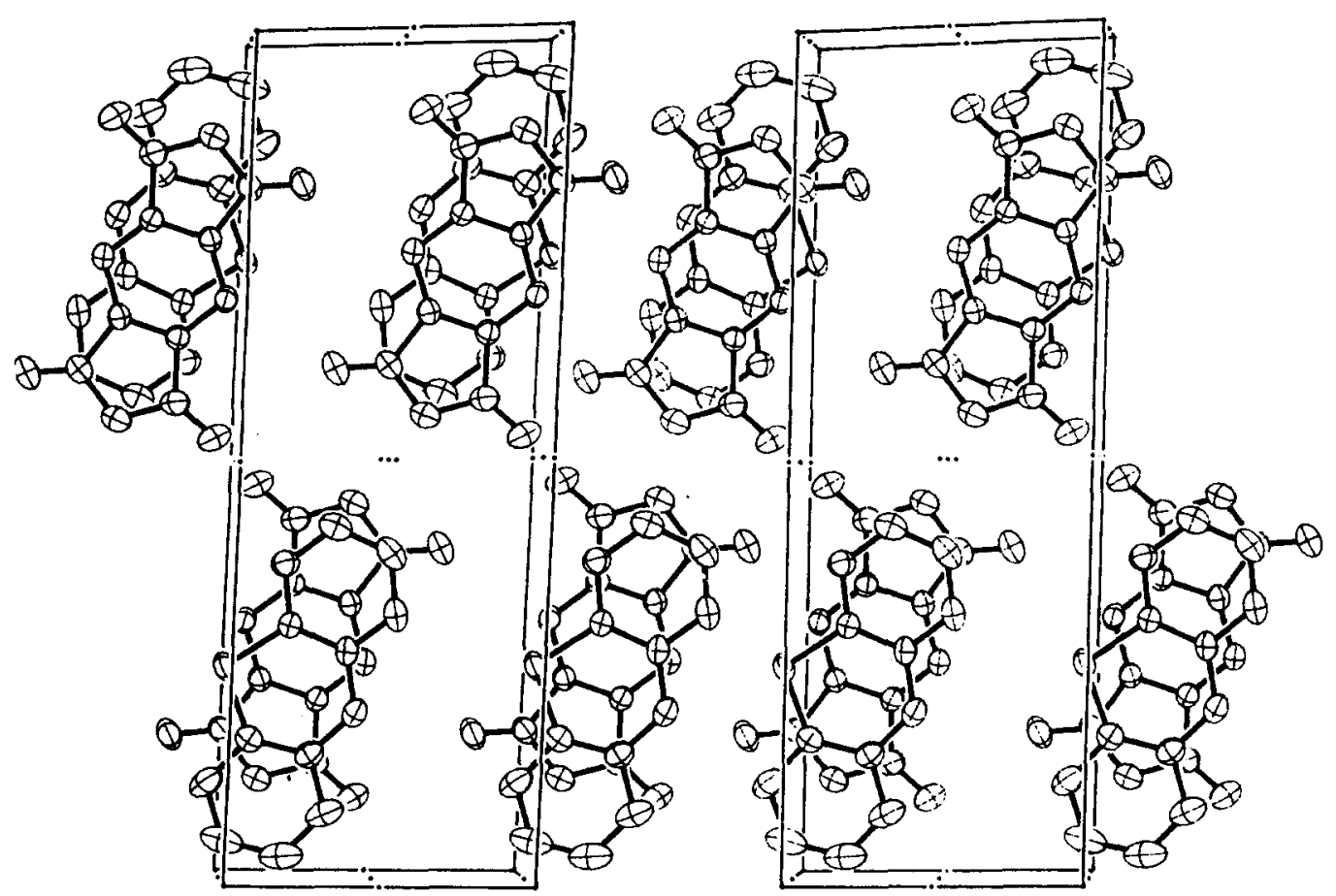

FIG. 4. Ste reoscopic packing diagram of PTZ: PMDA with the unit cell indicated. Inversion centers are presented as dots. The structure is projected down the $c$ axis (stack axis).

sites. This minor disorder differs from that reported for PTZ complexes with trinitrobenzene, ${ }^{17} \mathrm{TNB}$, and tetracyanoquinodimethane, ${ }^{18}$ TCNQ. In PTZ : PMDA, the enantiomer is packed with its $S$ and $N$ atoms interchanged. This packing defect allows the fold of the enantiomer to correspond to that expected by the symmetry of the lattice. The PTZ molecule in the complex with TNB has been described as either disordered with both enantiomers packing with their $\mathrm{N}-\mathrm{S}$ vectors oriented in the same direction or as undergoing dynamic folding. The author ${ }^{17}$ prefers the latter explanation and estimates the fold along the N-S axis to be from $165^{\circ}-$ $172^{\circ}$, considerably larger than in PTZ : PMDA. The PTZ : TCNQ complex displays a sinusoidal modulation, the period of which has been proposed ${ }^{18}$ to be approximately 4. 4(4) times the $b$ axis length. The PTZ molecule was planar within the limits of the precision possible in the analysis of the modulated structure. The disorder in the modulated PTZ : TCNQ structure appears to involve primarily a concerted shift in the center of gravity of adjacent columns and thus does not directly involve the question of planarity of the PTZ molecule but the resultant uncertainty in the atomic coordinates and thermal parameters could obscure modest nonplanarity.

The relatively rare occurrence of the packing error in PTZ : PMDA may be traceable at least in part to the weak hydrogen bond between the $\mathrm{N}-\mathrm{H}$ group of PTZ and atom $\mathrm{O}(13)$. There is no comparable interaction available to the enantiomer.

The fact that the data for this structure were collected from a crystal at room temperature has resulted in considerably larger thermal parameters than those in the A : PMDA structure determination ${ }^{7}$; see Fig. 6. Rigid body thermal motion calculations, ${ }^{19}$ Table II, using the nonhydrogen atoms of PMDA or PTZ or two "halves" of $\mathrm{PTZ}$ independently did not satisfactorily reproduce the observed thermal parameters. The possibility exists that the observed values contain systematic errors arising from the crystal size exceeding the region of homogeneity of the $\mathrm{x}$-ray beam. The bond distances in the PMDA moiety are sufficiently similar to those in the more accurate low temperature study of $A$ : PMDA to require no further comment.

We confine our comments concerning the bonding geometry of the PTZ moiety to the central ring which is expected to be subject to very little bond shortening as the result of thermal motion. The modest deviation of the PTZ moeity from planarity stands in contrast to noncomplexed phenothiazine. The dihedral angles between planes defined by the phenyl rings is $176.4^{\circ}$ in this structure which compares with $153.3^{\circ}$ in monoclinic phenothiazine ${ }^{8}$ and $158.5^{\circ}$ in orthorhombic phenothiazine. ${ }^{9}$ The singularity of the crystals of the latter form has been called into question ${ }^{20}$ but there is little reason to believe that the resultant bonding geometry from the crystal structure analysis is grossly in error. The $\mathrm{C}-\mathrm{S}$ and $\mathrm{C}-\mathrm{N}$ bond distances in this structure are very similar to those found in free phenothiazines; average values $1.758,1.770$, and $1.762 \AA$ for $\mathrm{C}-\mathrm{S}$ and $1.403,1.404$, and $1.399 \AA \mathrm{A}$ for $\mathrm{C}-\mathrm{N}$ in the three respective structures. Clearly the increased planarity of the PTZ moiety in this charge transfer complex has not been accompanied by the bond shortening expected if there were significantly greater $s$-orbital character in the $\mathrm{C}-\mathrm{S}$ and $\mathrm{C}-\mathrm{N}$ bonds of the planar conformer. The heteroatom bond angles are opened; the respective CSC values are $103.2^{\circ}, 99.6^{\circ}$, and $100.9^{\circ}$ and for CNC are 

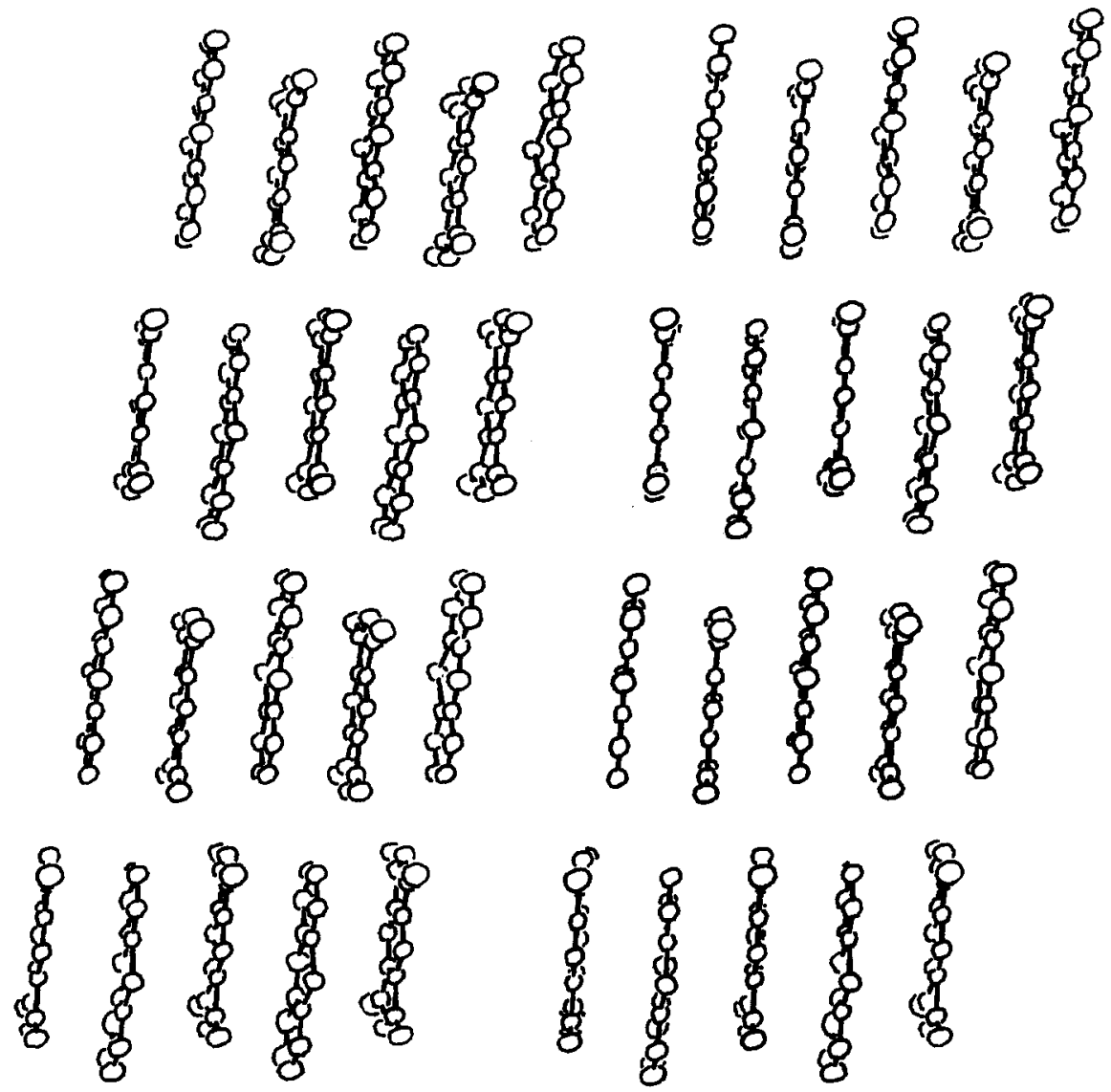

FIG. 5. A stereoscopic projection down the $a$ axis. The projection illustrates a pairwise interleaving of PTZ: PMDA stacks.

$125.3^{\circ}, 121.5^{\circ}$, and $124.4^{\circ}$. There is no evidence for increased aromaticity in the central ring of PTZ in this charge transfer complex. This is not surprising since a formal $2 e^{-}$transfer to the acceptor is required to achieve a $6 \pi e^{-}$structure for the ring.

Table III lists geometrical information relating the molecular orientation to the crystallographic axes. The angle between the normal to the plane of the central ring of the PMDA molecule and the stack axis ( $c$ axis) is $5.9^{\circ}$ which compares with an angle of $20.5^{\circ}$ in A: PMDA.

\section{OPTICAL PROPERTIES IN RELATION TO THE STRUCTURE}

\section{A. Dichroism, indicatrix}

Very thin layers of PTZ : PMDA crystals viewed through the (010) cleavage plane are strongly dichroitic in polarized light. Even our thinnest samples, several $\mu \mathrm{m}$ thick, appeared black when viewed with the $E$ vector parallel to [001]. However, with the orthogonal polarization, the thin samples became almost completely transparent whereas thicker samples appeared dark green. Since the absorption of the individual components begins in the violet (PTZ) and ultraviolet (PMDA), we conclude that in the visible region we are observing an intermolecular CT transition.

Next we want to establish the orientation of the indicatrix ${ }^{21}$ and the direction of the CT-transition dipole relative to it. The extinction directions of (010) platelets between crossed polarizers are very nearly $\left( \pm 3^{\circ}\right)$ parallel and perpendicular to the [001] axis; (because of dichroitic absorption they cannot be determined very precisely). When viewed conoscopically with a large aperture objective lens, one principal axis of the indicatrix is seen slightly off center. A symmetry plane appears perpendicular to [001] both for the system of the isogyres (using two crossed polarizers) and for the angular distribution of the dichroitic absorption (using one polarizer only). Consequently, another principal axis of the indicatrix as well as the CT-transition dipole moment must be nearly coincident with [001]. Since the crystal structure analysis has shown that [001] is the CT-stack axis, the PTZ : PMDA complex provides an example of the unusual case in which the direction of polarization of the CT-transition dipole is nearly coincident with one principal axis of the indicatrix. This structure therefore presents a considerably more favorable system for investigation of the CT transition than many other low symmetry systems with oblique stacking (such as A : PMDA) in which the $\mathrm{CT}$-transition dipole has major components along two or all three of the principal directions of the indicatrix.

From the minor deviations still present (in PTZ : PMDA) further structural conclusions can be drawn: The angular distribution of the dichroism looked at conoscopically through $(010)$ and with $E$ perpendicular to [001] reveals some residual $\mathrm{CT}$ absorption which has 

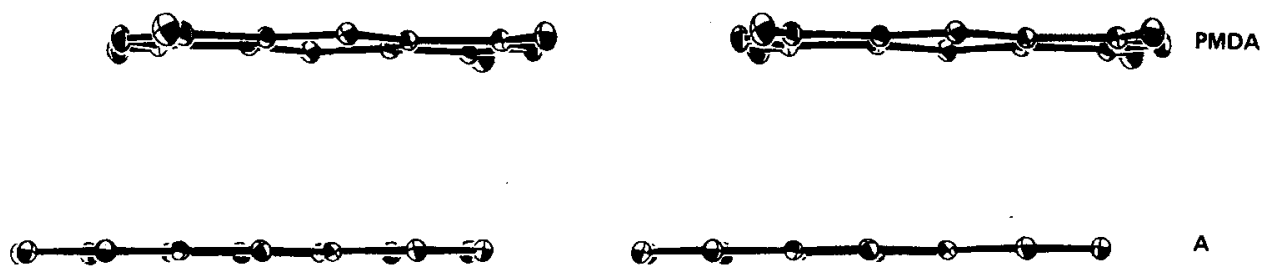

A
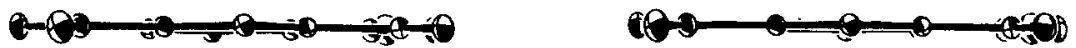

PMDA
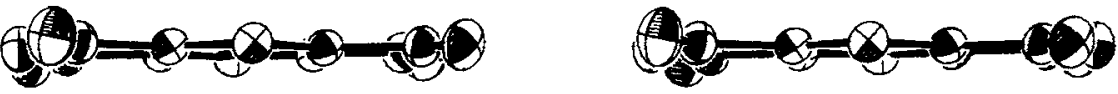

PMDA
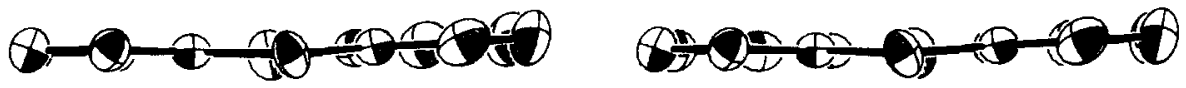

PTZ
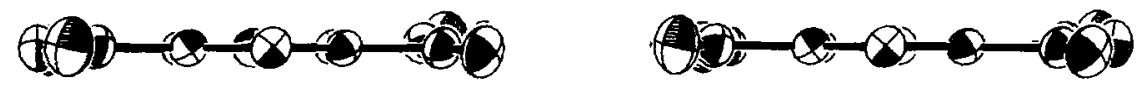

PMDA

FIG. 6. A comparison of the intrastack packing of anthracene: PMDA (6a) and PTZ: PMDA (6b).

its maximum for the wave vector $k$ in a direction that lies in the optical symmetry plane mentioned above, but is inclined to the perpendicular direction $b^{*}$. We attribute this observation to a modest deviation from parallelism between one of the principal axes of the indicatrix and the [001] intermolecular CT-transition dipole moment and/or to a minor transverse component in the CT-transition moment. Since the indicatrix contains contributions via Kramers-Kronig transform from all parts of the spectrum, and since the UV intramolecular optical transitions are bound to the symmetry of the individual molecules and in turn to the vectorial average of symmetry-related molecules, the first explanation leads immediately to a slightly inclined stacking. This conclusion is confirmed by the crystal structure analysis, which gives $5.9^{\circ}$ for the angle between the $c$ axis and the normal $\mathrm{N}$ to the plane of the PMDA molecule. The projection of this angle into the (010)

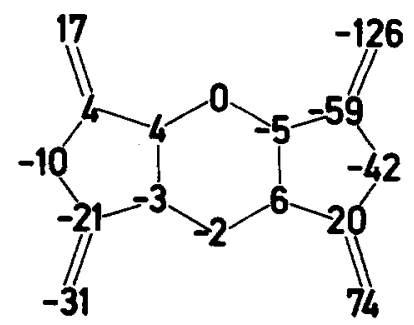

FIG. 7. The distance $(\AA \times 1000)$ of atoms of PMDA from a least squares plane (see Table III). The atom labels are the same as in Fig. 3(c, d). plane is $2.2^{\circ}$. The second explanation requires a zigzag stacking in which the molecular centers of gravity of the donors and acceptors are not colinear. This requirement is also fulfilled by the PTZ: PMDA structure.

Finally from the off $b^{*}, 1[001]$ direction of the principal axis seen conoscopically through $(010)$ one can conclude that one principal molecular polarizability and hence one (average) molecular axis lies in this oblique direction. This is also confirmed by the structure (Fig. 4).

\section{B. The CT-absorption spectrum}

The energy of the absorption edge of the singlet CT transition can be estimated from molecular data. For simplicity, let us neglect charge transfer in the ground state and assume complete charge transfer in the excited state. This is a good approximation for the systems under consideration here. We further restrict our selection to molecules of similar size, shape, packing properties, and polarizability. Under these conditions, the Coulomb interaction, $C$, of the electron and the hole separated by one donor-acceptor distance after a CT excitation will be similar in the systems compared and the lattice polarization will contribute comparable shifts. The only remaining individual quantities determining the $\mathrm{CT}$-transition energy, $E_{\mathrm{CT}}$, are the solid state ionization energy, $I$, of the donor and electron 
TABLE II. Rigid-body-motion parameters. The tensors are referred to the orthogonal coordinate system parallel to $a, c^{*} \times a$, and $c^{*}$. The origin is at the center of mass. Estimated standard deviations are given in parentheses.

\begin{tabular}{|c|c|c|c|c|c|c|c|c|}
\hline \multicolumn{3}{|c|}{$T\left(\AA^{2} \times 10^{4}\right)$} & \multicolumn{3}{|c|}{$L\left(\operatorname{rad}^{2} \times 10^{4}\right)$} & \multicolumn{3}{|c|}{$S\left(\AA \mathrm{Arad} \times 10^{4}\right)$} \\
\hline \multicolumn{9}{|l|}{ A. } \\
\hline \multirow[t]{3}{*}{$320(7)$} & $32(6)$ & $6(8)$ & $34(1)$ & $17(1)$ & $-1(1)$ & $-6(2)$ & $2(2)$ & $3(1)$ \\
\hline & $417(6)$ & $16(6)$ & & $56(3)$ & $-7(1)$ & $-3(3)$ & $11(4)$ & $5(2)$ \\
\hline & & $304(10)$ & & & $31(1)$ & $-3(1)$ & $2(1)$ & $0(22)$ \\
\hline
\end{tabular}

rms $\Delta U_{i j}=0.0019 \AA^{2}, \sigma\left(U_{i j}\right.$ calc $)=0.0021 \AA^{2}$, rms $\sigma\left(U_{i j}\right.$ obs $)=0.0006 \AA^{2}$.

B. Phenothiazine (Atoms 17 through 30)

$\begin{array}{rrrrrrrrr}373(19) & 73(14) & 9(20) & 33(4) & 25(6) & 2(3) & 0(6) & 0(7) & 19(3) \\ & 446(14) & 19(15) & & 78(15) & -2(5) & 1(11) & 1(12) & 3(6) \\ & & 344(32) & & & 48(2) & -44(3) & -22(4) & 0(50)\end{array}$

$\mathrm{rms} \Delta U_{i j}=0.0044 \AA^{2}, \sigma\left(U_{i j}\right.$ calc $)=0.0050 \AA^{2}$, rms $\sigma\left(U_{i j}\right.$ obs $)=0.0008 \AA^{2}$.

C. Part of phenothiazine centered in stack (atoms 17 through 20 and 23 through 26)

$\begin{array}{rrrrrrrrrr}365(6) & -6(5) & -23(7) & 75(3) & 24(4) & -12(2) & -2(4) & -37(4) & -4(2) \\ & 494(6) & 16(6) & & 73(6) & -3(3) & 0(4) & 14(4) & 6(2) \\ & & 311(11) & & & 51(2) & 42(2) & -39(2) & 0(14)\end{array}$

rms $\Delta U_{i j}=0.0010 \AA^{2}, \sigma\left(U_{i j}\right.$ calc $)=0.0014 \AA^{2}$, rms $\sigma\left(U_{i j}\right.$ obs $)=0.0006 \AA^{2}$.

D. Part of phenothiazine protruding from stack (atoms 17, 20, 21, 22, and 27 through 30 )

\begin{tabular}{|c|c|c|c|c|c|c|c|c|}
\hline \multirow[t]{3}{*}{$660(11)$} & $17(9)$ & $-23(12)$ & $70(5)$ & $-6(5)$ & $-9(4)$ & $4(7)$ & $34(6)$ & $48(4)$ \\
\hline & $441(1)$ & $26(11)$ & & $102(12)$ & $6(5)$ & $-4(7)$ & $-12(8)$ & $-5(4)$ \\
\hline & & $386(20)$ & & & $52(4)$ & $131(13)$ & $0(4)$ & $0(25)$ \\
\hline
\end{tabular}

$\operatorname{rms} \Delta U_{i j}=0.0019 \AA^{2}, \sigma\left(U_{i j}\right.$ calc $)=0.0025 \AA^{2}$, rms $\sigma\left(U_{i j}\right.$ obs $)=0.0009 \AA^{2}$.

affinity, $A$, of the acceptor; then

$$
E_{\mathrm{CT}}=I-A-C \text {. }
$$

In contrast to the ionization energy, the solid state electron affinity for most molecules is not known very accurately (c. $f_{0}$ Ref. 22). We have therefore chosen to eliminate the electron affinity of the acceptor and to avoid having to estimate the Coulomb energy, by combining two donors, $D_{1}$ and $D_{2}$, with the same acceptor:

$$
\begin{aligned}
& E_{1}^{\mathrm{CT}}=I_{1}-A-C \\
& E_{2}^{\mathrm{CT}}=I_{2}-A-C,
\end{aligned}
$$

which gives $E_{2}^{\mathrm{CT}}=E_{1}^{\mathrm{CT}}+\Delta$ where $\Delta=I_{2}-I_{1}$. The energy level diagram in Fig. 8 illustrates these relationships for donors $D_{1}=$ PTZ and $D_{2}=A$ with PMDA as the common acceptor. The value for $\Delta$ has been determined from data obtained from photoemission yield curves of the donor crystals: $I_{\mathrm{PTZ}}-I_{\mathrm{A}}=5.85-5.15=0.70 \mathrm{eV}$; see Fig. 9. The value $E_{1}^{\mathrm{CT}}$ for the singlet $S_{0}$ to singlet CT transition has been reported ${ }^{5}$ to be $2.27 \mathrm{eV}$ for A: PMDA; therefore the edge for the corresponding transition in PTZ : PMDA is expected at $E_{\mathrm{C}}=1.57 \mathrm{eV}$ which corresponds to $\lambda=789 \mathrm{~nm}$.

The CT-absorption spectra of a sublimation crystal polished down to a thickness of $130 \mu \mathrm{m}$ (area $\sim 1 \mathrm{~mm}^{2}$ ) for $k \| b^{*}$ and unpolarized light at different temperatures are plotted in Fig. 10. At $4.2 \mathrm{~K}$ there is-starting at $\sim 900 \mathrm{~nm}$-initially only a slow rise in the absorption coefficient with photon energy. Neither a sharp absorption edge nor indications of zero phonon lines are seen.
TABLE III. Least-squares planes and lines.

The $x, y$, and $z$ axes of the orthogonal coordinate system are in the directions $a, b^{*}$, and $a \times b^{*}$, respectively. In that coordinate system:

The plane of the central ring of the pyromellitic dianhydride molecule is given by

$0.2514 x-0.0944 y+0.9633 z=2.0257$.

The plane of the phenyl ring of phenothiazine formed by atoms $\mathrm{C}(18), \mathrm{C}(19), \mathrm{C}(23), \mathrm{C}(24), \mathrm{C}(25)$, and $\mathrm{C}(26)$ is given by

$0.2747 x-0.0951 y+0.9568 z=5.5113$.

The plane of the phenyl ring of phenothiazine formed by atoms $\mathrm{C}(21), \mathrm{C}(22), \mathrm{C}(27), \mathrm{C}(28), \mathrm{C}(29)$, and $\mathrm{C}(30)$ is given by

$$
0.2583 x-0.1558 y+0.9534 z=5.1762 \text {. }
$$
by

The line of the long axis of pyromellitic dianhydride is given

$$
\begin{aligned}
& x=2.7429+0.3575 t \\
& y=5.3471+0.9339 t
\end{aligned}
$$$$
z=1.8978+0.0027 t
$$

The terms of $a, b$, and $c$ and in terms of the unit vectors of the orthogonal coordinate system $\hat{i}, \hat{j}$, and $\hat{k}$, the unit normal to the central ring of the pyromellitic dianhydride molecule may be expressed as:

$$
\begin{aligned}
& N=-0.00533 a-0.00498 b+0.14733 c \\
& N=0.2514 \hat{i}-0.0944 \hat{j}+0.9633 \hat{k} .
\end{aligned}
$$

The angle between this normal and the $c$ axis is $5.9^{\circ}$. 
It is therefore difficult to locate the CT $S_{0} \rightarrow S_{1} 00$-transition. Quite obviously there is very strong electronphonon coupling with complete suppression of zero phonon lines. This is the very common case for DA complexes; the sharp zero phonon lines in A : PMDA are a remarkable exception. ${ }^{5}$

The absorption origin, Fig. 10, can be estimated somewhere around $900 \pm 25 \mathrm{~nm}$, i. e., $1.38 \mathrm{eV}$, a value that is $0.2 \mathrm{eV}$ less than expected. Accepting the validity of the assumptions made above, it is reasonable to attribute this result to a lower ionization energy of the PTZ molecule in the complex with PMDA than in the pure PTZ crystal. The decrease in ionization energy of complexed PTZ may be a result of the flattening of the normally bent PTZ molecule. (The ionization energy generally decreases with increasing extension of a conjugated $\pi$-electron system, c.f., Ref. 26a, Fig. 5.) The conformation revealed by the crystal structure determination of PTZ : PMDA is clearly more favorable for through conjugation of the $\pi$-electron system, though the bond distances do not indicate the onset of aromaticity.

\section{No optical emission}

Because the absorption edge is at approximately 900 $\mathrm{nm}$, any CT fluorescence or phosphorescence must be sought in the lower energy near IR region. To investigate the system for potential optical emission, crystals at temperatures between 4.7 and $300^{\circ} \mathrm{K}$ were excited with light of different wavelengths by irradiation with one of the following light sources: XBO 900, in combination with filters; HeNe laser at $632.8 \mathrm{~nm}, 5 \mathrm{~mW}$; krypton-argon laser at 488 and $514 \mathrm{~nm}, 400 \mathrm{~mW}$. No emission was detected. Subsequently an upper limit of the quantum yield was determined using a cooled S1 photomultiplier, a monochromater blazed at $1 \mu \mathrm{m}$, chopped light and lock-in detection. The upper limit was found to be less than $10^{-10}$ for the spectral region between 800 and $1100 \mathrm{~nm}$ under $10 \mathrm{~mW}$ excitation with $\lambda=514 \mathrm{~nm}$. Further, a relative quantum yield measurement was carried out which demonstrated that any emis-

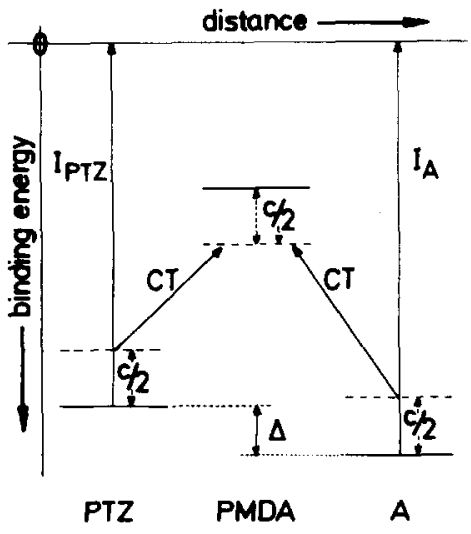

FIG. 8. Comparative ene rgy level diagram for the two DA complexes PTZ: PMDA and A : PMDA. Plotted is the one electron binding energy. The difference $\Delta$ of the energies of the $\mathrm{CT}$ transitions in the two complexes can essentially be traced to the ionization energies $I_{\mathrm{PTZ}}$ and $I_{\mathrm{A}}$ of the pure donor crystals $A$ and PTZ resp. $C / 2$ is half the Coulomb-binding energy of electron and hole in the ion pair formed by the CT transition.
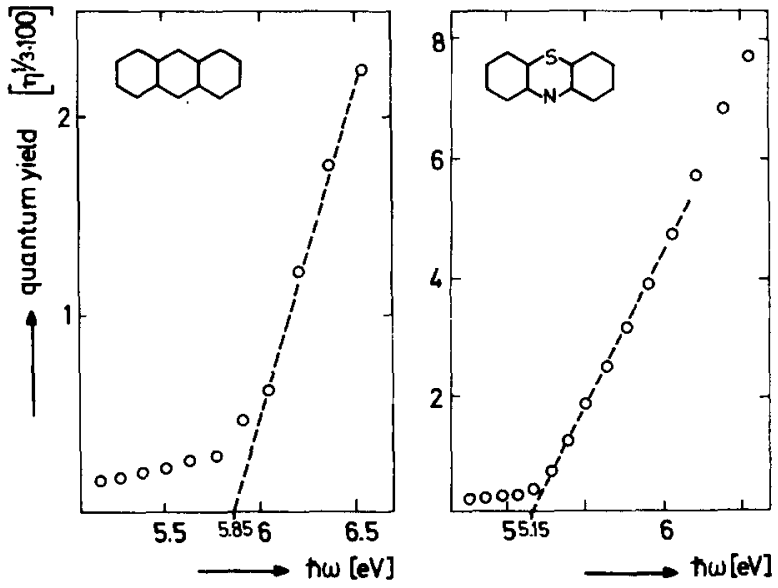

FIG. 9. Integral quantum yield $\eta$ of photoelectrons from $A$ and PTZ crystals as a function of the photon energy $\hbar \omega$. The threshold energy $E_{0}$ is extrapolated according to $\eta \alpha\left(\hbar \omega-E_{0}\right) .{ }^{3}$ (After Wache, Ref. 23.)

sion is at least a factor of $10^{5}$ less than that from A : PMDA.

In the energy region where CT emission would be expected, there may be combinations and overtones of molecular vibrations which could dissipate the excitation energy by radiationless transitions. The strongest coupling to the CT state will certainly be associated with the bending mode along the N... S axis with its concomitant change of the donator strength of the PTZ molecule and therefore of the CT energy. This mode will also be strongly coupled to the lattice phonons, where the periodic change of the static dipole moment of the PTZ molecule may play the dominant role.

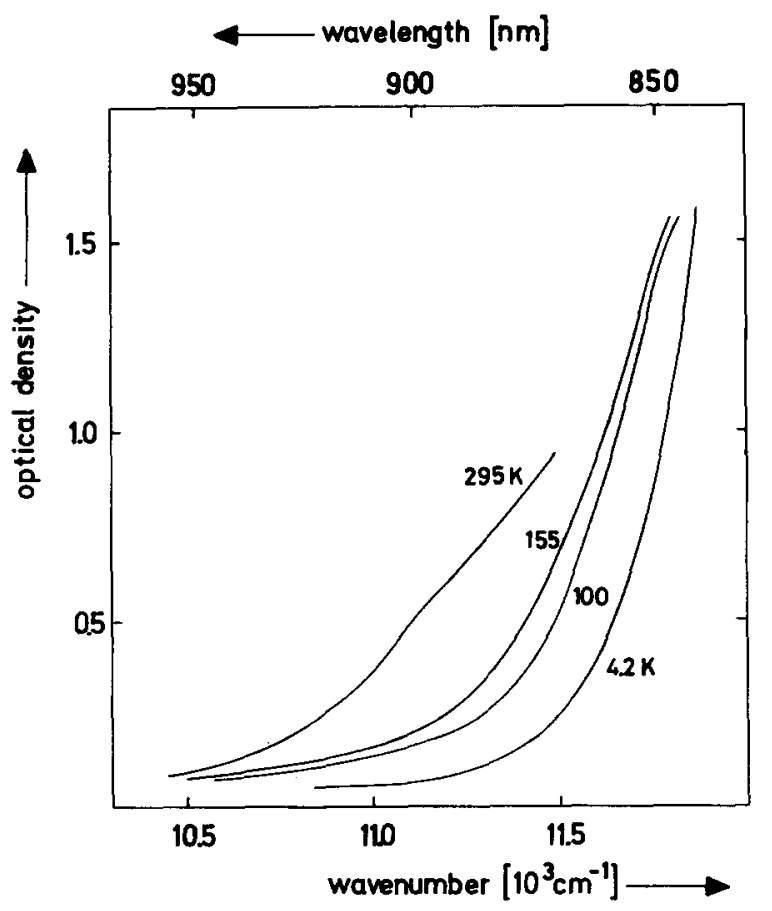

FIG. 10. Absorption edge of the CT transition in PTZ: PMDA at different temperatures. The crystal thickness was $130 \mu \mathrm{m}$. 


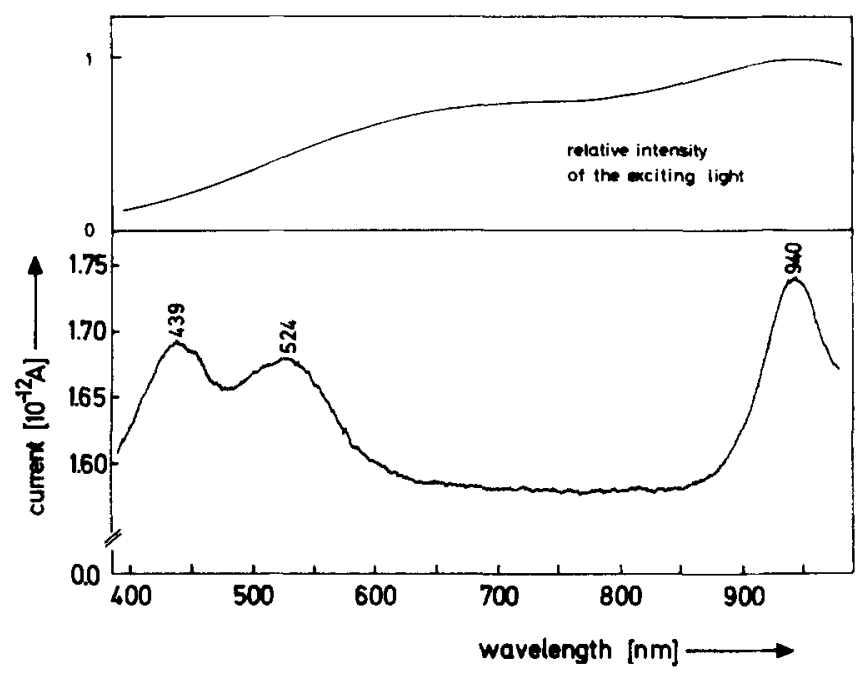

FIG. 11. Photocurrent excitation spectrum of PTZ : PMDA, not corrected for the intensity of the exciting light because of nonlinear light intensity dependence.

\section{GENERATION OF CHARGE CARRIERS}

Electrical measurements were performed with crystals contacted on opposite faces by semitransparent silver or opaque copper electrodes via vapor deposition or by painting with conductive silver paint. Measurements were performed under high vacuum. A tungsten halogen lamp $(650 \mathrm{~W})$ in combination with a Jarrel Ash $1 / 4 \mathrm{~m}$ monochromator was used for photocurrent excitation studies. Current measurements were made with a Cary 401 electrometer.

A photocurrent excitation spectrum is shown in Fig. 11. The three peaks therein can be associated with the region of the PTZ singlet absorption edge $(439 \mathrm{~nm})$, with the PTZ triplet $(524 \mathrm{~nm})$ and the region of CT absorption edge ( $940 \mathrm{~nm}$ or $1.32 \mathrm{eV})$. As in A : PMDA, the main photocurrent is generated just below the absorption edges in hot bands for which the penetration depth is comparable to the crystal thickness. This behavior is indicative of a high concentration of charge carrier traps which necessitates repeated detrapping steps across the volume.

In the absence of light we observe space charge perturbed dark currents with current voltage characteristics of $I \propto U^{n}(1.15 \leq n \leq 1.8$, from 50 to $5000 \mathrm{~V} / \mathrm{cm})$. The conductivity is $\sigma=10^{-12} \Omega^{-1} \mathrm{~cm}^{-1}$ for $E=2500 \mathrm{~V} / \mathrm{cm}$. The temperature dependence gives an activation energy of $E_{\text {act }}=0.85(5) \mathrm{eV}$.

By application of the formula for dark conductivity under intrinsic thermal carrier generation across a wide band gap into narrow bands with the dominating temperature dependence

$$
\sigma=\sigma_{\infty} \exp \left(-E_{\text {вad }} / 2 k T\right),
$$

we derive the thermal activation energy for this process to be:

$$
E_{\text {act }}=\frac{1}{2} E_{\text {gap }}
$$

giving $E_{\mathrm{eq}}=1.7 \mathrm{eV}$. In contrast to many other examples in the literature (pure anthracene is a very distinct example), the value of the activation energy obtained seems to reflect true band to band and not trap to band transitions: After the $1.38 \mathrm{eV}$ optical CT-transition, electron and hole are already separated by one DA distance, therefore the carriers need little additional energy to become completely free of their mutual Coulomb attraction. The difference of $0.32 \mathrm{eV}$ is a reasonable value for the ther mally activated step of final separation which means that each carrier needs $0.16 \mathrm{eV}$ of additional energy (Fig. 8). In A : PMDA an activation energy of the photoconduction of $0.14 \mathrm{eV}$ has been measured, ${ }^{2}$ which may be due to shallow trapping and therefore represent at least a lower limit for the activation energy of pair separation. From experimental activation energies for electron-hole separation in pure anthracene $\sim 0.5 \mathrm{eV}$ were derived for the pair binding energy of the nearest neighbor CT states. ${ }^{24}$ Electron-hole separation in anthracene/metal contacts needs $0.2 \mathrm{eV}$ for each carrier sign photoinjected into the anthracene crystals, c.f. Ref. 24, from which a pair binding energy of $\sim 0.4 \mathrm{eV}$ can be estimated. Calculations of electronhole interactions in anthracene also result in similar numbers. ${ }^{25}$ A more critical comparison would have to take into account that the electron can also jump, e.g. , to the left hand neighbor of the donor instead of jumping to the right hand neighbor (upper and lower in Fig. 6) leading to a more extended wave function in a double well potential with concomitant energy splittings, (see Haarer, Ref. 4).

We can estimate the dark concentration $C_{0}$ of thermally excited carriers based upon the gap energy and the assumption of a reasonable number for the density of states $N$. If we equate $N$ with the number of DA pairs per $\mathrm{cm}^{3}\left(2.25 \times 10^{21}\right)$, we find a carrier concentration $n=n^{+}+n^{-}=2.35 \times 10^{7} \mathrm{~cm}^{-3}$ at room temperature. If we further assume the mobilities $\mu^{+}, \mu^{-}$to be $1 \mathrm{~cm}^{2} \mathrm{~V}^{-1} \mathrm{~s}^{-1}$, (which is a typical value for charge carrier mobilities in organic molecular crystals $^{26,2}$ a dark conductivity of $\sigma=n e \mu=3.8 \times 10^{-12} \Omega^{-1} \mathrm{~cm}^{-1}$ results. This value is in remarkably good agreement with the measured value $\sigma=10^{-12} \Omega^{-1} \mathrm{~cm}^{-1}$ if one considers the exponential dependence of $C_{0}$ on $E_{\text {gap }}$ and the fact that the experimental current voltage characteristic is not ohmic, (indicating space charge).

Certainly, $10^{7}$ charge carriers per $\mathrm{cm}^{3}$ are not sufficient for detection by conventional ESR techniques. Indeed, only spurious signals were obtained. In view of the fact that the $\mathrm{CT}$-triplet energy must be less than the gap between the valence and conduction band one could be inclined to expect at least a detectable concentration of thermally created triplet excitons. However, since in this case only a single state is formed, rather than two independent particles, the full energy separation of ${ }^{\mathrm{CT}} T_{1}-S_{0} \sim{ }^{\mathrm{CT}} S_{1}-S_{0}=1.38 \mathrm{eV}$ appears in the exponent as activation energy, leading to a concentration of CTtriplet excitons which is even less than that of charge carriers.

\section{POLAR CT EXCITONS?}

As mentioned in the preceding section there is no preference for an electron to be excited to the right hand or 
left hand neighbor. In centro-symmetric : PMDA : A : PMDA : stacks and if no intrastack interactions are present, this is a typical symmetric double well potential problem leading to two degenerate levels with a symmetric and an antisymmetric wave function. Slightly different energies are expected in the presence of interactions. An optical dipole transition from the ground state is only allowed to one of the two states. ${ }^{4}$ Indeed, a zero field splitting was found for the 0 -phonon transition in A : PMDA. In an electric field, a Stark shift is observed, which mixes the states and thus gradually enhances (at the expense of the other) that transition that is forbidden in zero field.

We want to examine these ideas in view of the crystal structure of PTZ : PMDA. We note that there are polar stacks which, because of the inversion symmetry, are opposite each other in pairs. The polarity arises from the fact that all PTZ molecules within the same stack are bent in one direction. Therefore the dipole moments of the PTZ molecules add to give a single stack dipole moment (pyroelectric stacks). Despite the fact that the inversion center in the space-group precludes a macroscopic dipole moment, a CT exciton can be polar: To the extent to which interstack interaction can be neglected, the presence of the polar axis introduces directionality for the exciton. In particular, the two zero (external) field levels should be mixed by the (internal) intrastack dipole field and both should become allowed. This interesting effect can only be observed if the electron phonon coupling is weak enough to reveal sharp zero phonon lines. It is not yet clear, whether PTZ : PMDA is a system which can be brought to fulfill that requirement by careful handling, and measurement of crystals of the highest quality. However, by following ideas present in the literature ${ }^{5}$ one may eventually find a suitable system that combines polar stacks with zero phonon lines.

\section{ACKNOWLEDGMENTS}

The authors thank the Alexander von Humboldt Foundation of the Federal Republic of Germany and the $\mathrm{Na}$ tional Research Council of Canada for the award of a Humboldt Fellowship and a Travel Fellowship to B. E. Robertson, the Universität Stuttgart and the University of Regina for assistance and the National Research Council of Canada for support in the form of an operating grant. The collaboration of Dr. H. Schulz and K. H. Thiemann with $R$. A. for the determination of initial lattice constants and crystal forms is gratefully acknowledged. The authors are indebted to Dr. P. Fischer of the Institut für Organische Chemie, Biochemie and Isotopenforschung of the Universität Stuttgart for chemical elementary analysis.

\footnotetext{
${ }^{1}$ D. Haarer and N. Karl, Chem. Phys. Lett. 21, 49 (1973).

${ }^{2}$ N. Karl and J. Ziegler, Chem. Phys, Lett. 32, 438 (1975).

${ }^{3}$ C. J. Eckhardt and J. Merski, Surf. Sci. 37, 937 (1973).
}

${ }^{4}$ D. Haarer, Chem. Phys. Lett. 27, 91 (1974); ibid. 31, 192 (1975); D. Haarer, M. R. Philpott, and H. Morawitz, J. Chem. Phys. 63, 5238 (1975).

${ }^{5}$ D. Haarer, J. Chem. Phys. 67, 4076 (1977).

${ }^{6} \mathrm{~J}$. Ziegler and N. Karl, Chem. Phys. 40, 207 (1979).

${ }^{7}$ B. E. Robertson and J. J. Stezowski, Acta Crystallogr. Sec. B 34, 3005 (1978).

${ }^{8}$ J. D. Bell, J. F. Blount, O. V. Briscoe, and H. C. Freeman, Chem. Commun. 1968, 1656.

9 J. J. H. McDowell, Acta Crystallogr. Sec. B 32, 5 (1976).

${ }^{10}$ Determined by mass spectrometry, N. Karl (unpublished).

${ }^{11} \mathrm{~N}$. Karl, 1st Europ. Conf. on Crystal Growth, Zürich (1976), abstract book p. 80; 5th Internat. Conf. on Cryst. Growth, Boston (1977), abstract book p. 207; see also N. Karl, Habilitationsschrift (University of Stuttgart, Germany, 1975).

${ }^{12}$ J. M. Stewart, P. A. Machin, C. S. Dickinson, H. L. Ammon, H. Heck, and H. Flack, The X-ray System, Version 1976 (University of Maryland, Computer Science Center, College Park, MD., Technical Report TR-446, 1976), Unless otherwise indicated this computer program library was used for all calculations.

${ }^{13}$ W. R. Busing, K. O. Martin, and H. A. Levy, ORFLS, Report ORNL-TM-305 Oak Ridge National Laboratory, Tennessee (1962). The scattering factors other than for the hydrogen atoms were from D. T. Cromer and J. B. Mann, Acta Crystallogr. Sec. A 24, 321 (1968). Those for hydrogen atoms were from R. F. Stewart, E. R. Davidson, and W. T. Simpson, J. Chem. Phys. 42, 3175 (1965).

${ }^{14} \mathrm{~J}$. P. Malrieu and B. Pullman, Theor. Chim. Acta 2, 293 (1964).

15'See AIP document no. PAPS-JC PSA-71-1244-33 for 33 pages of observed and calculated structure factors. Order by PAPS number and journal reference from American Institute of Physics, Physics Auxiliary Publication Service, 335 East 45th Street, New York, N. Y. 10017. The price is $\$ 1.50$ for each microfiche (98 pages), or $\$ 5$ for photocopies of up to 30 pages with $\$ 0.15$ for each additional page over 30 pages. Airmail additional. Make checks payable to the American Institute of Physics."

${ }^{16}$ C. K. Johnson, ORTEP-II, Report ORNL-5138, Oak Ridge National Laboratory, Tennessee (1971).

${ }^{17} \mathrm{C}$. J. Fritchie, Jr., J. Chem. Soc. A 1969, 1328.

${ }^{18}$ H. Kobayashi, Acta Crystallogr. Sec. B 30, 1010 (1973).

${ }^{19}$ V. Schomaker and K. N. Trueblood, Acta Crystallogr. Sec. B 24,63 (1968).

${ }^{20}$ B. W. Van de Waal and D. Feil, Acta Crystallogr. Sec. B 33, 314 (1977).

${ }^{21}$ The indicatrix of absorbing crystals is complex. However for the small oscillator strength of the CT transition, it is a good approximation to neglect the small ellipticity of the wave propagation modes and to restrict the argumentation to the real part.

${ }^{22}$ E. C. M. Chen and W. E. Wentworth, J. Chem. Phys. 63, 3183 (1975).

${ }^{23} \mathrm{R}$. Wache, Diplomarbeit (University of Stuttgart 1973).

${ }^{24} \mathrm{H}$. Baessler and H. Killes reiter, Mol. Cryst. Liq. Cryst. 24,21 (1973).

${ }^{25}$ A. Jurgis and E. A. Silinsh, Phys. Status Solidi B 53, 735 (1972); E. A. Silinsh, Electronic States of Organic Molecular Crystals (Zinantne, Riga, 1977) (in Russian).

${ }^{26}$ Experimental data on charge carrier mobilities in organic molecular crystals are collected, e.g., in (a) N. Karl, "Organic Semiconductors," in Advances in Solid State Physics (Pergamon, New York, 1974), p. 261; (b) L. B. Schein, Phys. Rev. B 15, 1024 (1977); (c) W. Warta, Diplomarbeit (University of Stuttgart, Germany, 1978). 